[5] R. T. Lauer, P. H. Peckham, K. L. Kilgore, and W. J. Heetderks, "Application of cortical signals to neuroprosthetic control: A critical review," IEEE Trans. Rehab. Eng., vol. 8, pp. 205-208, 2000.

[6] G. Pfurtscheller, C. Guger, G. Müller, G. Krausz, and C. Neuper, "Brain oscillations control hand orthosis in tetraplegic," Neurosci. Lett., vol. 292, no. 3, pp. 211-214, 2000.

[7] D. M. Taylor, S. I. H. Tillery, and A. B. Schwartz, "Direct cortical control of 3D neuroprosthetic devices," Science, vol. 296, pp. 1829-1832, June 2002.

[8] A. P. Georgopoulos and J. T. Massey, "Cognitive spatial-motor processes: 2. Information transmitted by the direction of two-dimensional arm movements and by neuronal populations in primate motor cortex and area 5," Experiment. Brain Res., vol. 69, pp. 315-326, 1988.

[9] A. P. Georgopoulos, A. B. Schwartz, and R. E. Ketter, "Neuronal population coding of movement direction," Science, vol. 233, pp. 1416-1419, 1986.

[10] C. Shannon and W. Weaver, The Mathematical Theory of Communication. Urbana, IL: Univ. Illinois Press, 1949.

[11] F. Rieke, D. Warland, R. de Ruter van Steveninck, and W. Bialek, Spikes: Exploring the Neural Code, 2nd ed, T. J. Sejnowski and T. A. Poggio, Eds. Cambridge, MA: MIT Press, 1997, pp. 101-126.

[12] P. H. Peckham, M. W. Keith, K. L. Kilgore, J. H. Grill, K. S. Wuolle, G. B. Thrope, J. Hobby, M. J. Mulcahey, S. Carroll, V. R. Hentz, and A. Wiegner, "Efficacy of an implanted neuroprosthesis for restoring hand grasp in tetraplegia: A multicenter study," Archives Phys. Med. Rehab. vol. 82, no. 10 , pp. 1380-8, Oct. 2001.

[13] P. M. Fitts, "The information capacity of the human motor system in controlling the amplitude of movement," J. Experiment. Psych., vol. 47, pp. 381-391, June 1954.

\section{Multimodal Neuroelectric Interface Development}

Leonard J. Trejo, Kevin R. Wheeler, Charles C. Jorgensen, Roman Rosipal, Sam T. Clanton, Bryan Matthews, Andrew D. Hibbs, Robert Matthews, and Michael Krupka

\begin{abstract}
We are developing electromyographic and electroencephalographic methods, which draw control signals for human-computer interfaces from the human nervous system. We have made progress in four areas: 1) real-time pattern recognition algorithms for decoding sequences of forearm muscle activity associated with control gestures; 2) signal-processing strategies for computer interfaces using electroencephalogram (EEG) signals; 3) a flexible computation framework for neuroelectric interface research; and d) noncontact sensors, which measure electromyogram or EEG signals without resistive contact to the body.
\end{abstract}

Index Terms-Brain-computer interfaces (BCI), electroencephalogram (EEG), electric field sensors, electromyogram (EMG), neuroelectric interfaces.

\section{INTRODUCTION}

We define a system that couples the human nervous system electrically to a computer as a neuroelectric interface-a sensing and

Manuscript received July 15, 2002; revised February 3, 2003.

L. Trejo, K. R. Wheeler, C. C. Jorgensen, R. Rosipal, and B. Matthews are with the NASA Ames Research Center, Moffett Field, CA 94035 USA (e-mail: 1trejo@mail.arc.nasa.gov; kwheeler@mail.arc.nasa.gov; cjorgensen@ mail.arc.nasa.gov; rrosipal@mail.arc.nasa.gov; bmatthews@mail.arc.nasa. gov).

S. Clanton is with OEIC, Worthington, OH 43085 USA (e-mail: sclanton@oeic.net).

A. D. Hibbs, R. Matthews, and M. Krupka are with Quantum Applied Science and Research, Inc., San Diego, CA 92121 USA (e-mail: andy@quasarusa.com; robm@quasarusa.com).

Digital Object Identifier 10.1109/TNSRE.2003.814426 processing system that can use signals from the brain or from other parts of the nervous system, such as peripheral nerves, to achieve device control. We regard brain-computer interfaces (BCIs) [1] as a subset of neuroelectric interfaces. Our current focus is on using features from electroencephalograms (EEGs) and electromyograms (EMGs) as control signals for various tasks, such as aircraft or vehicle simulations and other graphic displays.

Our long-term goals are to: 1) develop new modes of interaction that cooperate with existing modes such as keyboards or voice; 2) augment human-system interaction in wearable, virtual, and immersive systems by increasing bandwidth and quickening the interface; and 3) enhance situational awareness by providing direct connections between the human nervous system and the systems to be controlled. Our near-term goals include: 1) a signal acquisition and processing system for real-time device control; 2) automatic EMG-based recognition and tracking of human gestures; and c) feasibility testing of EEG-based control methods.

In this paper, we will survey selected results and demonstrations of EMG- and EEG-based neuroelectric interfaces. We will describe an EMG-based flight stick, an EMG-based numeric keypad, an EEGbased interface for smooth, continuous control of motion in a graphic display, and comparison of algorithms for modeling the EEG patterns associated with real and imagined hand motion. Finally, we will discuss recent developments of noncontact electric field sensors for EMG and EEG recording.

Our approach is to describe a body of developmental research, which is still in progress, and to indicate methods that have potential for engineering development. Given the BCI focus of this Special Issue, descriptions of purely EMG-based interfaces will be brief. We will describe the EEG results and the new sensor developments in more detail.

\section{EMG INTERFACES}

\section{A. EMG-Based Flight Stick}

In our first demonstration, a computer transformed EMG signals recorded from four bipolar channels placed on the forearm of a subject into control signals for an aircraft simulator. Thus, the processed EMG signals controlled an imaginary flight stick [2]. EMG samples were processed in real time using a flexible signal-processing framework developed in our laboratory. Our feature extraction procedures included routines to filter out redundant and meaningless channels with a mutual information metric [3]. The features were moving averages of the EMG signal from overlapping windows, where the data within a window are nearly stationary. ${ }^{1}$ Our model for mapping EMG signal features to gestures uses mixtures of Gaussians within a hidden Markov model context. We tested and validated this system with many trials over a two-year period in three subjects, who flew and landed high-fidelity simulations of a Boeing F-15 Eagle or a Boeing 757-200 freighter aircraft. Control of both aircraft was adequate for normal maneuvers. For the 757, a real-time landing sequence under neuroelectric control was filmed at NASA Ames Research Center (see on-line demos [4] and [5]).

\section{B. EMG-Based Numeric Keypad}

We have also found that EMG signals from the arm can distinguish typing of one key from another on a "virtual keyboard." In this demon-

\footnotetext{
${ }^{1}$ We used overlapping moving averages of the rectified, unfiltered EMG signal, sampled at either 500 (joystick task) or $2000 \mathrm{~Hz}$ (typing task). The windows contained 128 points and overlapped preceding windows by 96 points. We tried other types of features such as autoregressive coefficients, wavelets, and short-time Fourier transforms, but the moving averages provided the most robust response for everyday use.
} 
stration, we programmed a computer to translate eight bipolar EMG signals recorded from the forearm into commands for typing the digits 0-9 on a virtual numeric keypad. We used the same processing system for the typing interface as for the EMG flight stick (see footnote ${ }^{1}$ ). However, the hidden Markov model was retrained using EMG data recorded during typing. Tests were performed with random lists of data to be entered. The typing activity consisted of using the numeric keypad on a computer keyboard. One participant was trained and used for the experiments. He was allowed to type $0-9$ and "enter." In tests, the subject typed the series 1-9 and 0 three times, or a series of four different dates consisting of four digits each. Using such lists, we found that the digits 0-9 could be detected with $100 \%$ accuracy from the processed EMG signals. A demonstration of this system was also recorded at NASA Ames Research Center [see on-line demo [6]].

\section{EEG INTERFACES}

\section{A. One-Dimensional Graphic Device Control}

Previous research has shown that control signals for graphic devices, such as cursors, can be drawn from EEG signals such as $\mu$ and $\beta$ rhythms [7]. Our approach is to develop a flexible processing system, which will adapt to different tasks and users. To do this, we explored several tasks and an array of pattern recognition and machine learning algorithms.

In open-loop tests, we measured EEG during real or imaginary motions and analyzed motion-related changes in the EEG signals later. The tasks were mouse motion and keyboard typing. In closed-loop tests, we used a real-time system, which provided visual feedback about motion-related EEG signals to the user. The feedback algorithms included narrow-band linear filters for signals such as $\mu$-rhythm, broadband filters developed adaptively, on-line measures of EEG complexity, and support vector machine classifiers [8], [9]. In one closed-loop task, the subject moved a needle gauge up or down by voluntary control of EEG. We trained subjects with a series of target needle positions. In another task, we mapped the subject's EEG signals to left and right turns of a simulation of a Mars rover vehicle as it traveled at constant speed over a Mars terrain database. In both tasks, subjects viewed either the gauge or the rover on a large video display.

In each task, either 32 or 64 channels of EEG were recorded with a QuickCap (Neuromedical Supplies, Inc.) using the extended International 10-20 System [10] with digitally linked mastoid references (1000-Hz sampling rate, $1-30-\mathrm{Hz}$ bandpass). We visually inspected the multichannel EEG recordings and hand-selected artifact-free segments that clearly contained oscillatory activity. On these segments, we used singular value decomposition (SVD) to reduce the multichannel recordings to a small number of SVD components. Generally, from four to eight SVD components were sufficient to account for $95 \%$ of the variance in the hand-selected EEG segments. In some experiments, we approximated these components with a few electrodes, located near the positive and negative extremes of the scalp distributions of the SVD loadings. For these recordings, we used either a 2-channel EEG headset (Sensorphone, Allied Products, NY) or disposable self-adhesive Ag-Cl electrodes (Neuromedical Supplies, VA). For example, for the mouse motion task in Subject 1, we approximated the first two SVD components with electrode pairs FC1-TP7 and AF8-P4, respectively. For the mouse motion task in Subject 2, we used a set of 12 electrodes that formed two lines straddling $\mathrm{Cz}$ and parallel to the interaural line, with all electrodes uniformly spaced $4 \mathrm{~cm}$ apart. ${ }^{2}$

\footnotetext{
${ }^{2}$ In all EEG tests, we ruled out EMG contamination of the EEG signals as a source of control. We computed the average event-related band power of several narrow bands between 0.1 and $50 \mathrm{~Hz}$ for both EEG and EMG signals in a 1-s long interval, centered on motion onset. For all bands above $5 \mathrm{~Hz}$, EMG power in the band increased during the motion, whereas EEG power either decreased or remained unchanged.
}

In Subject 1, a 45-year old right-handed male, open-loop tests showed that $\mu$-rhythm bursts were visible in the raw EEG. The $\mu$-rhythm spectral peak was centered at $9 \mathrm{~Hz}$. A narrow-band $6-11-\mathrm{Hz}$ filter was satisfactory for closed-loop feedback. Using the smoothed filter output power, Subject 1 was able to drive the needle gauge up or down to reach target locations in two testing sessions. In Subject 2, a 32 year-old left-handed male, $\mu$-rhythms were not visible in the raw EEG.

Since a filter for $\mu$-rhythm was not clearly satisfactory for both subjects, we explored other, more general measures of EEG complexity. The idea here is that regardless of the specific peaks at which sensorimotor EEG rhythms oscillate, their synchrony will influence signal complexity. In our context, we define complexity as a measure reflecting changes in EEG regularity or predictability. Signals corresponding to periods of high EEG synchrony will be more regular, predictable, and will have low complexity. Periods of relatively low EEG synchrony will have high complexity.

We examined coarse-grained entropy rates (CER), Gaussian process entropy rates (GPER), spectral entropy (SE), and wavelet entropy (WE). CER is an empirical complexity measure based on stochastic process entropy rates and the Kolmogorov-Sinai entropy of nonlinear dynamical systems [11], [12]. CER have been shown to reflect complexity of physiological signals [10], [13], [14]. If we consider the EEG to be a zero-mean stationary Gaussian process, we can estimate entropy rates directly from the EEG spectrum [15], [16]. Thus, we define GPER to be a linear measure, which can fully describe an underlying stationary Gaussian process but cannot describe nonlinear data. SE is a measure which computes Shannon entropy over the normalized power spectral density function; i.e., periodogram [17]. There is a clear connection between GPER and SE as both measures reflect changes of the frequency spectra of the EEG over different brain states. For WE, we extend the concept of SE by replacing the Fourier transform with the discrete wavelet transform [18], [19]. So for WE, we computed Shannon entropy over the wavelet coefficients at individual resolution levels.

We applied these measures to open loop data from the mouse motion task in the two subjects. For each measure, estimates were computed for the first two SVD components over time in windows of 2048 samples, which were advanced in 100-sample steps, and further smoothed with a 9-point noncausal running mean (Fig. 1). For Subject 1, three measures - SE, GPER, and CER — reflected changes in EEG synchrony or complexity at nearly the same times as the $\mu$-rhythm. The WE measure correlated poorly with $\mu$-rhythm. For component 1 , the correlation coefficients of each measure with $\mu$-rhythm in the real-motion condition were $r=-0.87,-0.87,-0.87$, and -0.62 , for SE, GPER, CER, and WE, respectively. The corresponding correlations for the imaginary condition were $r=-.85,-0.81,-0.79$, and -0.55 . All correlations were significant ( $t$-test, $p<0.001$ ). Tests of Spearman rank-order correlations produced the same results, but with lower values of $r(-0.49$ to -0.75 ). Qualitatively similar results were obtained for Subject 2. We completed several real-time tests and demonstrations of EEG-based control of the Mars rover using complexity measures. For Subject 2, we recorded a demonstration of one of the sessions in which the CER served as the control signal [on-line demo, [20]].

\section{B. EEG-Based Typing}

For the EEG-based typing tasks, we sought to detect the periods of physical keyboard typing activity from EMG-free EEG recordings and to use linear models or machine-learning algorithms to translate the EEG signals into interface commands. We did not seek to identify which keys were pressed. We sought to discriminate typing from rest and also to discriminate left- from right-hand typing. Our approach was the same as for the motion control tasks: multichannel EEG recordings were reduced to a few SVD components. These components served as 


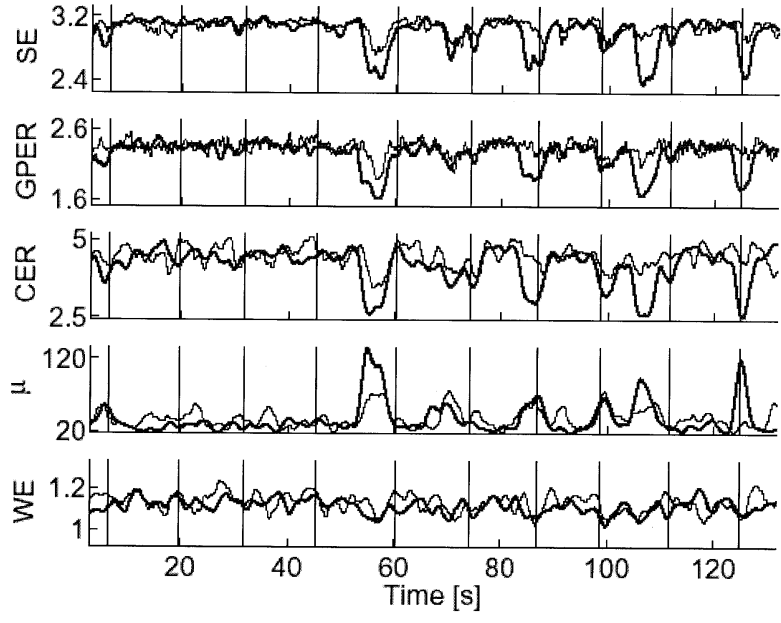

(a)
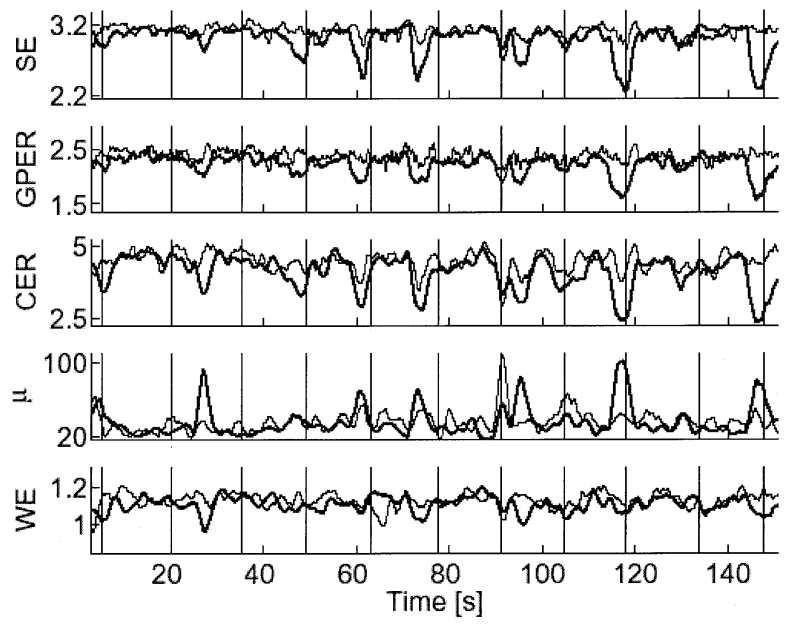

(b)

Fig. 1. $\mu$-rhythm filter performance compared with estimates of EEG complexity for the open-loop mouse motion task in Subject 1. Lines show the filter outputs for a series of EEG input windows over time. The heavy line is for SVD component 1, which was derived from earlier 32-channel recordings and estimated here with bipolar electrodes FC1-TP7. The thin line is for SVD component 2, estimated by AF8-P4. Vertical lines mark the times when real or imagined hand motions began. Both components change near the onset of real or imagined motions, but component 1 shows larger and more regular changes than component 2 . The $\mu$-rhythm filter has increased output power, or synchronization, before motions and decreased power, or desycnhronization, during or after motions. The other measures (SE, GPER, CER, and WE) estimate signal complexity, which change in a direction opposite to that of $\mu$-rhythm. For a description of each measure, see the text. (a) Real left-hand movement. (b) Imaginary left-hand movement.

inputs to filters or algorithms that tracked typing behavior. We used the same two subjects who performed the mouse motion tasks and a third subject, a 47-year old right-handed male. Our results are limited to open-loop tests with real motion, and do not imply that classification of EEG is possible without the motor task.

For both subjects, we collected six 5-min runs consisting of typing the keys $A$ or $F$ with the left pinkie and index fingers, or typing the keys $J$ or ; (semicolon) with the right index and pinkie fingers, or alternating use of the left and right hands within a single run. Typing consisted of self-paced bursts of keystrokes lasting about $5 \mathrm{~s}$ followed by about $10 \mathrm{~s}$ of rest. In other tests, subjects pressed a single key and then rested for about $10 \mathrm{~s}$. The EEG was sampled at $1000 \mathrm{~Hz}$, digitally band-passed from 1 to $30 \mathrm{~Hz}$, and resampled at $100 \mathrm{~Hz}$. EMG data from the left and right forearms were recorded with four pairs of electrodes placed on the wrists and upper forearms. EMG was sampled at $1000 \mathrm{~Hz}$, digitally band-passed from 30 to $150 \mathrm{~Hz}$, then resampled at $300 \mathrm{~Hz}$ and rectified. To model typing behavior using EEG, we tested the following three different types of algorithms:

2) $\mu$-Rhythm filter: a linear finite impulse response (FIR) filter with a passband centered on the peak of $\mu$-rhythm signals observed near electrodes $\mathrm{C} 3$ or $\mathrm{C} 4$ in the subject's resting EEG;

3) Adaptive linear combiner $(A L C)$ : the Widrow-Hoff LMS algorithm [21] was used to model periods of the EMG signal corresponding to rest using the EEG time series;

4) Support vector classifier (SVC): we used the LIBSVM software for linear support vector classification [22], [23].

We found that the $\mu$-rhythm filter was inadequate to model the relationship between EEG and periods of typing or rest. We next explored modeling typing and rest segments with an ALC. Here, we found that for Subject 1, a 50-tap ALC was sufficient to track the motion and rest periods associated with typing. For Subject 2, who had no clear $\mu$-rhythm, a 500-tap ALC also tracked rest and typing. The results suggest that EEG signals associated with typing can serve as an index of the typing activity. A previous report using a different task drew a similar conclusion [24].

With an ALC, it is possible to freeze adaptation after training and plot the spectrum of the transfer function (Fig. 2). For Subject 1, both the 50and 500-tap filters converged to a set of simple, unimodal transfer functions that favored frequencies below $10 \mathrm{~Hz}$. For Subject 2, the transfer functions appeared to be bimodal, with one broad peak in the $5-10-\mathrm{Hz}$ range and another broad peak in the $10-15-\mathrm{Hz}$ range. In the 500 -tap filters for Subject 2, a third broad peak is present in the $20-25-\mathrm{Hz}$ range.

The ALCs were trained to use EEG-SVD component inputs to model EMG activity exclusively during rest periods. So the ALC output is higher during the periods of rest than during typing. Thus, the filter output serves as a rest detector, or conversely, the filter error serves as a motion detector. For Subject 1, the 50-tap filter produced higher output during rest than during typing (Fig. 3). For Subject 2 (not shown), the 500-tap filter performed in a similar fashion.

For the conditions in which typing consisted of single keystrokes followed by rest, the ALC filters (and some nonlinear variants) did not serve well for discriminating typing from rest. To solve this problem, we attempted to classify EEG segments as either motion or nonmotion using windowed EEG signals as inputs to a SVC. Subject 3 performed five 5-min runs of single-key typing. In each of the first four runs, the subject typed for a few seconds using either the left or right hand then rested for a few seconds. In the fifth run, the subject alternated between right- and left-hand typing with rest periods in between. Filtered, 64-channel EEG signals served as inputs to the SVC. The data were digitally low-pass filtered at $30 \mathrm{~Hz}$ and down sampled to $128 \mathrm{~Hz}$. Successive 128-point segments (1 s of data with 75\% overlap) were labeled as nonmotion, left-hand motion, or right-hand motion. Periods were classified as motion when the mean of the corresponding left- or right-hand EMG signal was greater than a predefined threshold. A linear SVC was trained on EEG signals from either the odd- or even-numbered runs and tested on the remaining runs. SVC results for left- versus right-hand typing were inconclusive, with accuracies near $60 \%$. However, the SVC's successfully classified motion versus nonmotion with accuracies between $78 \%$ and $91 \%$. In the most general case-training with all four initial runs and testing with the final run of mixed hand motion - the classification accuracies for rest versus left-hand typing, rest versus right-hand typing, or rest versus either hand typing were $85 \%, 82 \%$, and $88 \%$, respectively. Increasing the EEG bandwidth by refiltering with a $64-\mathrm{Hz}$ cutoff did not substantially change these values (respectively: 84\%, 87\%, and 87\%), suggesting that EMG artifact did not contribute to the classification.

We also analyzed the weights derived using linear SVC as we did for the ALCs. A spectral analysis of the support vector weights revealed a prominent peak at $18 \mathrm{~Hz}$, which was well defined over centro-parietal 


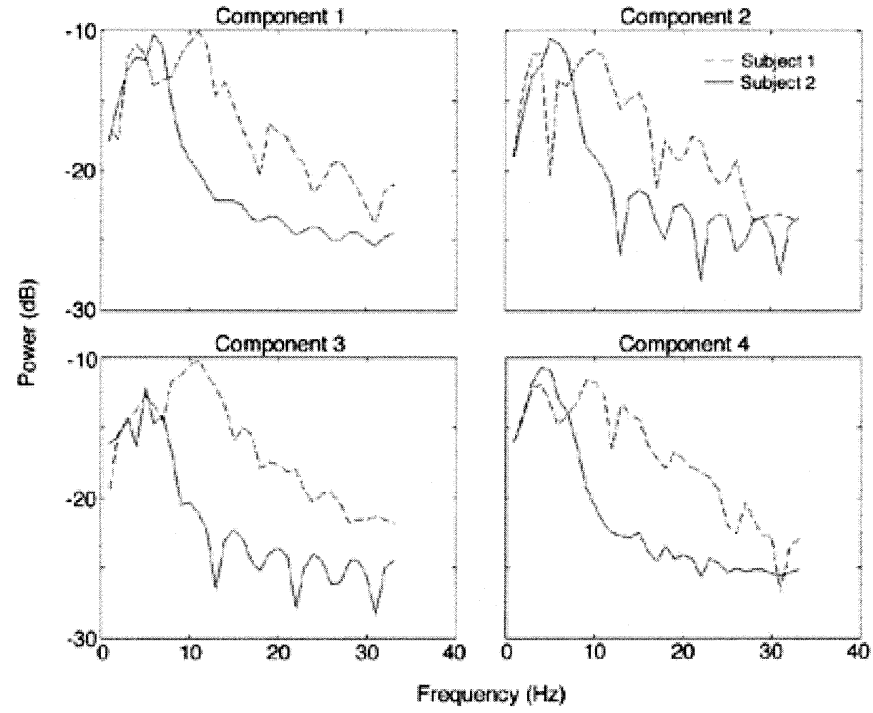

(a)

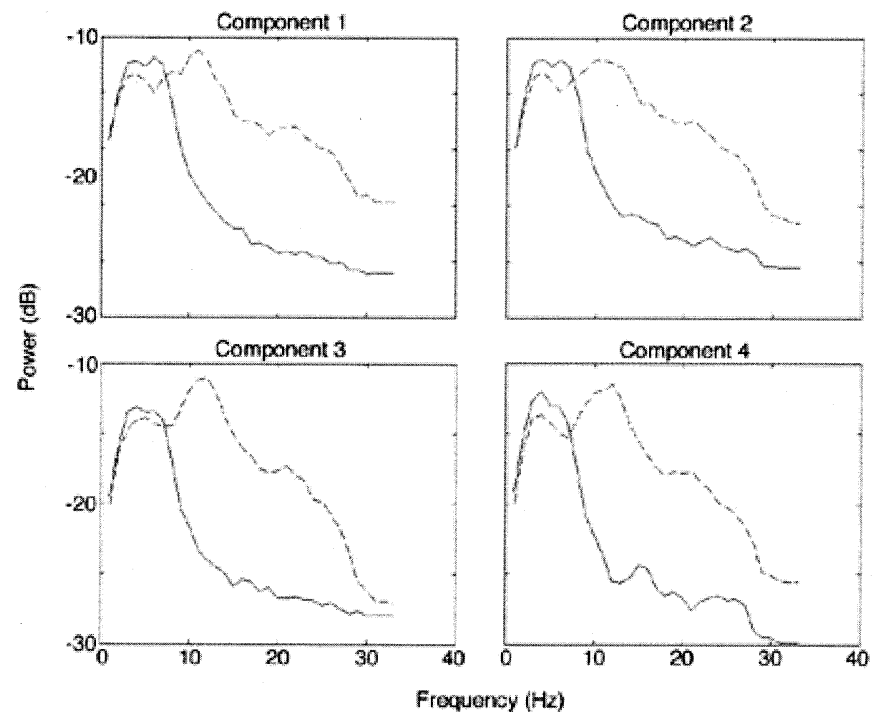

(b)

Fig. 2. Transfer functions of the (a) 50-tap and (b) 500-tap ALCs trained to predict rest or right-hand typing periods for the first four SVD EEG components. Typing consisted of bursts of alternating keystrokes using pinkie and index fingers for a few seconds, followed by a few seconds of rest.

electrodes $\mathrm{C} 1, \mathrm{C} 2, \mathrm{Cp} 1$, and $\mathrm{Cp} 2$. We found in a separate analysis that reducing the 64-channels to six channels, including these four, F1 and F2 allowed for classification accuracy of $90 \%$ for the test in which the four initial runs served as training data for the fifth run.

\section{Noncontact SENSOR DEVElopMENT}

The NASA Ames Research Center is working with Quantum Applied Science and Research, Inc. (QUASAR) to develop new sensors for neuroelectric recordings. These sensors can measure the electric potential in free space and so they do not require resistive, or even good capacitive coupling to the subject. The principal sensor innovation is providing high-input impedance for the electrode that senses the free space potential, while accommodating the input bias current of the amplifier. The input capacitance of present electrometer grade amplifiers is of order $1-3 \mathrm{pF}$. This allows us to arrange the coupling capacitances of the electrometer to yield a near ideal measurement of the bioelectric potential.

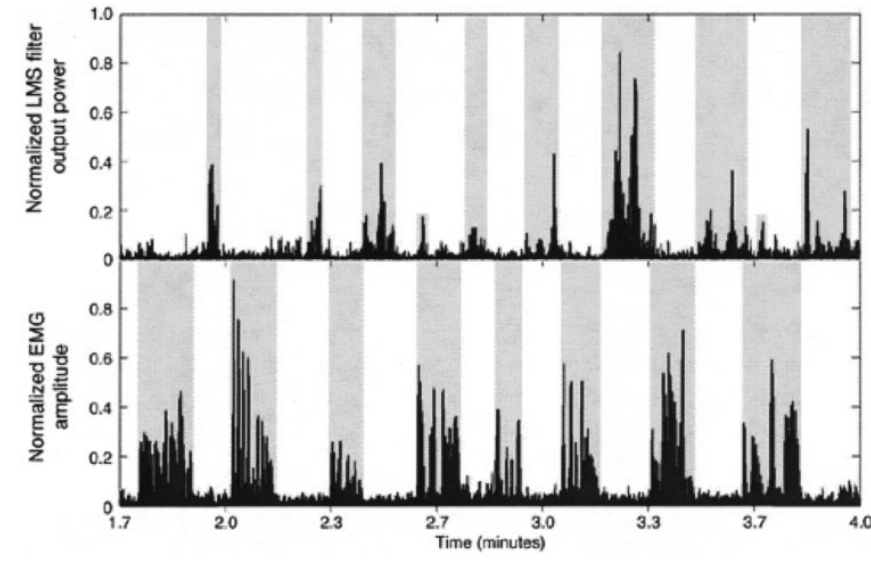

Fig. 3. Performance of the ALC on test data in Subject 1 over time, showing tracking of the motion and rest periods. The upper graph shows the filter output; the lower graph shows the rectified EMG time series. Shaded regions correspond to continuous left-hand finger tapping (lower) or rest (upper). Since the filter was trained to model the EEG signals during the rest periods, periods of motion correspond to relatively low filter output power. This is because the EEG signals during motion are different than during rest, and so do not match the filter derived for EEG signals during rest periods.

Despite its small size, the new sensor is approximately 100 times better than prior electric potential sensors [25]. At $10 \mathrm{~Hz}$, it has comparable sensitivity to conventional resistive contact (dry or wet) electrodes. In the off-body mode, the sensor can make an accurate measurement through clothing. The sensor also has a broadband response from 0.01 to $10 \mathrm{kHz}$, proving sufficient bandwidth to measure EEG and EMG, and essentially all other bioelectric signals of interest.

In our initial tests, we have made direct comparisons between surface recordings of EMG and EEG with noncontact recordings of the same signals.

\section{A. EMG Tests}

We recorded EMG from two surface $\mathrm{Ag}-\mathrm{AgCl}$ electrodes spaced 2 $\mathrm{cm}$ apart on the forearm over the flexor carpi radialis. The subject was asked to make a fist and this signal was recorded for multiple trials. Then these wet electrodes were removed and replaced by a QUASAR noncontact $E$-field sensor and the subject repeated the fist clenching exercise. The noncontact sensor recordings tracked the conductive electrode EMG recordings well in the range from 500 to $2000 \mathrm{~Hz}$.

\section{B. EEG Tests}

We recorded EEG from eight surface $\mathrm{Ag}-\mathrm{AgCl}$ electrodes spaced 4-cm apart and lying on lines $2-\mathrm{cm}$ anterior or posterior to $\mathrm{Cz}$, running from left to right, all referred to average mastoids with ground at AFz. A QUASAR noncontact $E$-field sensor was tested at the points lying in between the EEG electrodes. EEG was recorded with a Neuroscan Nuamp at gain of 19, bandpass noncontact sensor tracked the main features of the EEG spectrum seen in the $\mathrm{Ag}-\mathrm{AgCL}$ electrode recordings (Fig. 4). For example, both recordings show a clear peak in the spectrum near $10 \mathrm{~Hz}$, which reflects endogenous alpha rhythm.

\section{DISCUSSION AND CONCLUSION}

The EMG-based joystick and typing tasks were chosen to replicate something with which computer users are already familiar. These traditional types of interfaces are certainly not suitable for gesture-based systems as they force unnatural and unintuitive movements. Signal processing and machine learning are maturing to a point whereby methods such as hidden Markov models are suitable for ordinary laptops without 


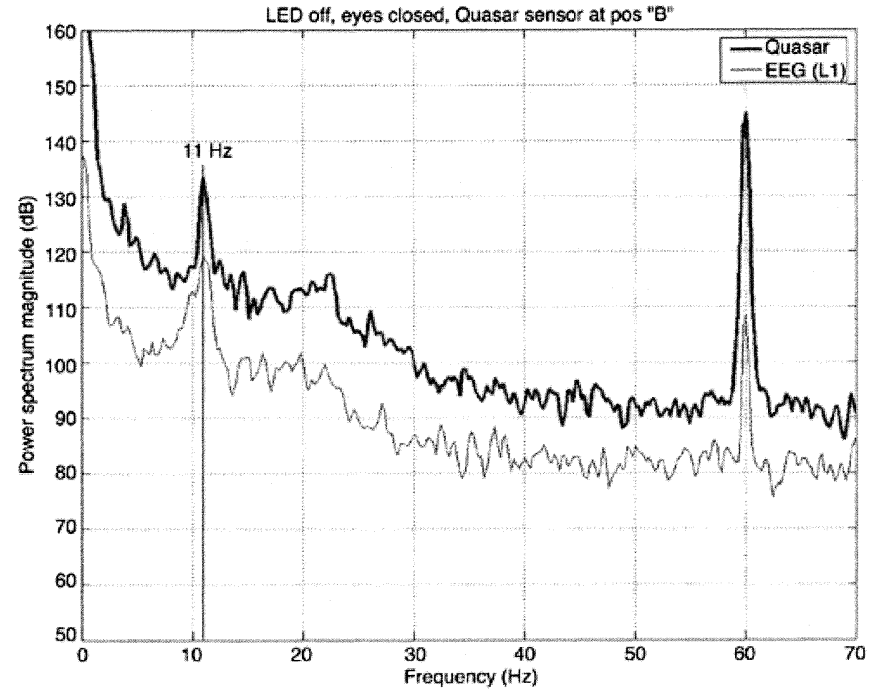

Fig. 4. Power spectrum of recordings from QUASAR and $\mathrm{Ag}-\mathrm{Cl}$ electrodes in a 21-year-old male subject. The Quasar sensor tracks the main features of EEG spectrum seen in the Ag-AgCL electrode recordings. Including the peak near $10 \mathrm{~Hz}$, which reflects endogenous alpha rhythm. The line at $60 \mathrm{~Hz}$ is noise from the main power lines resulting from imperfect shielding.

special hardware, however the user interfaces are still two-dimensional mouse-based systems. The ultimate trial of our EMG methodology will be to have a system with a more natural gesture command interface. This could then be used to test the performance of EMG-based systems for everyday use by regular users. Once multiple users have been run on multiple tasks we will then be able to form a usability assessment.

Our EEG-based developments show that one-dimensional (1-D) control of a graphic device is feasible as a human computer interface. For different subjects different algorithms may be required, such as $\mu$-rhythm filters or complexity measures. Our system is programmed to allow rapid switching among these algorithms or parallel use of the algorithms. We have demonstrated control of a needle gauge and a rendition of turning a Mars rover simulator left and right in real time.

We found that the type of task and the qualities of EEG in a subject interact with the signal processing requirements of the interface. In the simplest case, a narrow bandpass filter tracked 1-D continuous motion for a subject with clear $\mu$-rhythm. Other, more general measures, such as SE, GPER, and CER tracked continuous motion in a subject who did not have a clear $\mu$-rhythm. Our data sample is too limited to allow us to assess the relative discriminative power of the various measures-we can only show that several measures, which use the full EEG spectrum, provide information similar to that given by a $\mu$-rhythm filter. Such measures could be useful in a wider range of subjects, especially those who do not have a clear $\mu$-rhythm. In the typing tasks, more elaborate filters, such as an ALC or the SVC-derived filters were required. As the complexity of the task increased from 1-D motion to typing with different fingers on right and left hands, we found that increasing amounts of data and algorithmic complexity were required. For the single-key typing task, as many as 8192 coefficients (64 channels $\times 128$ samples) were used in the SVC. However, we also found that analysis of the SVC weights could reduce the number of channels from 64 to 6 (or 768 coefficients) without sacrificing accuracy. In any case, the computational demands for using these algorithms in real-time are modest and do not pose a barrier to applications.

At least two serious limitations apply to our data. First, the number of subjects is small. This was necessary to allow us time to explore a wide range of algorithms. Second, our experiments are qualitative and lack statistical and quantitative metrics, such as bit rate, as used in other
BCI studies. For the present, we must present these results as merely being indicative of promising BCI approaches for device control.

Our initial findings with the QUASAR noncontact sensors show that it is possible to record both EMG and EEG signals of high fidelity without a conductive link to the body. The bandwidth and gain of these sensors are appropriate for practical applications.

\section{ACKNOWLEDGMENT}

The authors wish to thank C. Curry and M. Allen of QSS Group, Inc., who helped to program the software for the demonstrations. They also appreciate the helpful comments of two anonymous reviewers.

\section{REFERENCES}

[1] J. R. Wolpaw, N. Birbaumer, W. J. Heetderks, D. J. McFarland, P. H. Peckham, G. Schalk, E. Donchin, L. A. Quatrano, C. J. Robinson, and T. M. Vaughan, "Brain-computer interface technology: A review of the first international meeting," IEEE Trans. Rehab. Eng., vol. 8, pp. 164-173, June 2000.

[2] C. Jorgensen, K. Wheeler, and S. Stepniewski, "Bioelectric control of a 757 class high fidelity aircraft simulation," in Proc. World Automation Congr., Wailea, Maui, HI, June 11-16, 2000.

[3] J. Bilmes, "Maximum mutual information based reduction strategies for cross-correlation based joint distributional modeling," in Proc. ICASSP, Seattle, WA, Apr. 1998, pp. 469-472.

[4] "Twitching instead of clicking" (2001). [Online]. Available: http://abcnews.go.com/sections/wnt/DailyNews/wnt_flightechnology010 513. html

[5] "Extension of the Human Senses Group," Ames News, (2001). [Online]. Available: http://ic.arc.nasa.gov/projects/ne/ehs.html

[6] "Extension of the Human Senses Group. EMG Video," Ames News, (2001b). [Online]. Available: http://ic.arc.nasa.gov/projects/ ne/videos/NECD320x240.3.mov

[7] J. R. Wolpaw et al., "An EEG-based brain-computer interface for cursor control," Electroencephalogr. Clin. Neurophysiol., vol. 78, pp. 252-259, 1991.

[8] B. Schölkopf and A. J. Smola, Learning with Kernels. Cambridge, MA: MIT Press, 2002.

[9] V. Vapnik, Statistical Learning Theory. New York: Wiley, 1998.

[10] H. Jasper, "The ten-twenty electrode system of the international federation," Electroencephalogr. Clin. Neurophysiol., vol. 43, pp. 397-403, 1958.

[11] M. Paluŝ, "Coarse-grained entropy rates for characterization of complex time series," Physica D, vol. 96, pp. 64-77, 1996.

[12] T. M. Cover and J. A. Thomas, Elements of Information Theory. New York: Wiley, 1991.

[13] M. Paluŝ, V. Komárek, Z. Hrnĉîr, and T. Procházka, "Is nonlinearity relevant for detecting changes in EEG?," Theory Biosci., vol. 118, pp. 179-188, 1999.

[14] R. Rosipal, "Kernel-based regression and objective nonlinear measures to assess brain functioning," Ph.D. dissertation, Univ. of Paisley, Paisley, U.K., 2001.

[15] S. Ihara, Information Theory for Continuous Systems. Singapore: World Scientific, 1993.

[16] M. Paluŝ, "On entropy rates of dynamical systems and Gaussian processes," Phys. Lett. A, vol. 227, pp. 301-308, 1997.

[17] T. Inouye, K. Shinosaki, H. Sakamoto, S. Toi, S. Ukai, A. Iyama, Y. Katsuda, and M. Hirano, "Quantification of EEG irregularity by use of the entropy of the power spectrum," Electroencephalogr. Clin. Neurophysiol., vol. 79, pp. 204-210, 1991.

[18] S. Blanco, A. Figliola, R. Q. Quiroga, O. A. Rosso, and E. Serrano, "Time-frequency analysis of electroencephalogram series (III): Information transfer function and wavelets packers," Phys. Rev. E, Stat. Plasmas Fluids Relat. Interdiscip. Top., vol. 57, pp. 932-940, 1998.

[19] R. Q. Quiroga, O. A. Rosso, E. Basar, and M. Schürmann, "Wavelet entropy in event-related potentials: A new method shows ordering of EEG oscillations,” Biol. Cybern., vol. 84, pp. 291-299, 2001.

[20] EHS Group. EEG Quicktime Video (2002). [Online]. Available: http: //ic.arc.nasa.gov/projects/ne/videos/EHS-RR-MARS-ROVER-060 402. MOV

[21] B. Widrow and S. D. Stearns, Adaptive Signal Processing. NJ: Prentice-Hall, 1985. 
[22] C.-C. Chang and C.-J. Lin, "Training nu-support vector classifiers: Theory and algorithms," Neural Comput., vol. 13, pp. 2119-2147, 2001.

[23] C.-W. Hsu and C.-J. Lin, "A comparison of methods for multi-class support vector machines," IEEE Trans. Neural Networks, vol. 13, pp. 415-425, Mar. 2002

[24] T. Mima, T. Matsuoka, and M. Hallen, "Information flow from cortex to muscle in humans," Clin. Neuorphysiol., vol. 112, pp. 122-126, 2001.

[25] D. D. Sentman, "Schumann resonances," in Handbook of Atmospheric Electrodynamics, H. Volland, Ed. Boca Raton, FL: CRC, 1995.

\section{The Wadsworth Center Brain-Computer Interface (BCI) Research and Development Program}

\section{Jonathan R. Wolpaw, Dennis J. McFarland, Theresa M. Vaughan,} and Gerwin Schalk

\begin{abstract}
Brain-computer interface (BCI) research at the Wadsworth Center has focused primarily on using electroencephalogram (EEG) rhythms recorded from the scalp over sensorimotor cortex to control cursor movement in one or two dimensions. Recent and current studies seek to improve the speed and accuracy of this control by improving the selection of signal features and their translation into device commands, by incorporating additional signal features, and by optimizing the adaptive interaction between the user and system. In addition, to facilitate the evaluation, comparison, and combination of alternative BCI methods, we have developed a general-purpose BCI system called BCI-2000 and have made it available to other research groups. Finally, in collaboration with several other groups, we are developing simple BCI applications and are testing their practicality and long-term value for people with severe motor disabilities.
\end{abstract}

Index Terms-Augmentative communication, brain-computer interface (BCI), conditioning, electroencephalography (EEG), mu rhythm, rehabilitation, sensorimotor cortex.

\section{INTRODUCTION}

In awake people, primary sensorimotor cortical areas often display 8-12-Hz electroencephalographic (EEG) activity when not engaged in processing sensory input or producing motor output [1]-[3], (reviewed in [4]). This idling activity—called mu rhythm when focused over somatosensory or motor cortex, and visual-alpha rhythm when focused over visual cortex-is thought to be produced by thalamocortical circuits [4], [5]. Mu-rhythm activity comprises a variety of different $8-12-\mathrm{Hz}$ rhythms and is usually associated with $18-26-\mathrm{Hz}$ beta rhythms [6]-[9]. Mu and beta rhythms wax and wane in association with actual movement or imagination of movement [9]-[12].

In our brain-computer interface (BCI) studies, people with or without motor disabilities (e.g., amyotrophic lateral sclerosis, cerebral palsy, spinal cord injury) learn to control mu- and/or beta-rhythm

Manuscript received January 31, 2003; revised April 25, 2003. This work was supported by the National Center for Medical Rehabilitation Research of the National Institute of Child Health and Human Development under NIH Grant HD30146, by the National Institute of Biomedical Imaging and Bioengineering under NIH Grant EB00856, and by the ALS Hope Foundation.

The authors are with the Laboratory of Nervous System Disorders, Wadsworth Center, New York State Department of Health and State University of New York, Albany, NY 12201 USA (email: wolpaw@wadsworth.org; mcfarlan@wadsworth.org; vaughan@wadsworth.org; Schalk@wadsworth.org).

Digital Object Identifier 10.1109/TNSRE.2003.814442 amplitudes to move a cursor in one or two dimensions to choices on a computer screen [13]-[15]. Fig. 1(a) illustrates the basic phenomenon. In this example, the user controls vertical cursor movement by controlling the amplitude of a $12-\mathrm{Hz}$ mu rhythm focused over left sensorimotor cortex. The frequency spectra indicate that control is focused in the mu-rhythm band and to a lesser extent in a beta-rhythm band.

In our standard protocol, a linear equation translates mu-rhythm or beta-rhythm amplitude from one or several scalp locations into cursor movement 10 times/s. Users learn over a series of 40-min sessions to control the cursor. They participate in 2-3 sessions per week, and most demonstrate significant control within 2-3 weeks. In initial sessions, users typically employ some form of motor imagery (e.g., imagination of hand movements, whole body activities, relaxation, etc.) to control the cursor. As training proceeds, imagery usually becomes less important, and users report that they move the cursor just as they perform normal movements, that is, without thinking about the details of performance.

While EEG from only one or two scalp locations control the cursor online, data from 64 locations over the entire scalp (recorded with an electrode cap) are stored for subsequent offline analysis. This analysis defines the full topography of EEG changes associated with target position, detects non-central nervous system (CNS) artifacts such as electromyographic (EMG) or electrooculographic (EOG) activity, and helps guide improvements in online operation. It relies largely on the measure $\mathrm{r}^{2}$, the proportion of the total variance in mu- or beta-rhythm amplitude that is accounted for by target position and thereby reflects the user's level of EEG control. For example, the $\mathrm{r}^{2}$ topographical analysis in Fig. 1(a) shows that control is sharply focused over left sensorimotor cortex and in the mu- and beta-rhythm frequency bands. This measure correlates well with the accuracy of target selection, and, thus, can be used in offline analysis to identify alternative signal features that are likely to improve performance [16].

With this control, users can move the cursor to answer spoken yes/no questions with accuracies $>95 \%$ [17], [18]. They can also achieve independent control of two different mu- or beta-rhythm channels and use that control to move a cursor in two dimensions [19]. Recent work has concentrated on developing precise one-dimensional control, and on applying it to choosing among up to eight different selections. Users have achieved information transfer rates up to $20-25 \mathrm{~b} / \mathrm{min}$ [20], [21].

\section{CurRent Aims}

Our research has concentrated on defining the topographical, spectral, and temporal features of mu- and beta-rhythm control and on optimizing the mutually adaptive interactions between the user and the BCI system. Our central goal is to improve the speed and accuracy of BCI communication and to show that it can serve the practical needs of people with severe motor disabilities. In accord with this goal, we are focusing on four major aims.

\section{A. Optimizing Feature Selection, Extraction, and Translation}

We are evaluating alternative methods for selecting and extracting the signal features, that is, the mu- and beta-rhythm amplitudes that control cursor movement. This evaluation includes assessments of additional signal processing methods, recording locations, and frequency bands. For example, we have found that the choice of spatial filtering method is critically important. For mu and beta rhythms, a common average reference or a large (6- $\mathrm{cm}$ interelectrode distance) Laplacian 